\title{
Clinical Study \\ Repeat Whole Brain Radiation Therapy with a Simultaneous Infield Boost: A Novel Technique for Reirradiation
}

\author{
William A. Hall, Roshan S. Prabhu, Ian R. Crocker, Anees Dhabban, Tomi Ogunleye, \\ Shravan Kandula, Xiaojun Jiang, Walter J. Curran, and Hui-Kuo G. Shu \\ Department of Radiation Oncology and the Winship Cancer Institute, Emory University, 1365 Clifton Road, NE, \\ Suite CT-104, Atlanta, GA 30322, USA \\ Correspondence should be addressed to Hui-Kuo G. Shu; hgshu@emory.edu
}

Received 7 October 2013; Accepted 18 March 2014; Published 26 May 2014

Academic Editor: Oliver Micke

Copyright (c) 2014 William A. Hall et al. This is an open access article distributed under the Creative Commons Attribution License, which permits unrestricted use, distribution, and reproduction in any medium, provided the original work is properly cited.

\begin{abstract}
The treatment of patients who experience intracranial progression after whole brain radiation therapy (WBRT) is a clinical challenge. Novel radiation therapy delivery technologies are being applied with the objective of improving tumor and symptom control in these patients. The purpose of this study is to describe the clinical outcomes of the application of a novel technology to deliver repeat WBRT with volume modulated arc therapy (VMAT) and a simultaneous infield boost (WB-SIB) to gross disease. A total of 16 patients were initially treated with WBRT between 2000 and 2008 and then experienced intracranial progression, were treated using repeat WB-SIB, and were analyzed. The median dose for the first course of WBRT was 35 Gy (range: 30-50.4 Gy). Median time between the initial course of WBRT and repeat WB-SIB was 11.3 months. The median dose at reirradiation was 20 Gy to the whole brain with a median boost dose of $30 \mathrm{~Gy}$ to gross disease. A total of 2 patients demonstrated radiographic disease progression after treatment. The median overall survival (OS) time from initial diagnosis of brain metastases was 18.9 months (range: 7.1-66.6 (95\% CI: 0.8-36.9)). The median OS time after initiation of reirradiation for all patients was 2.7 months (range: 0.46-14.46 (95\% CI: 1.3-8.7)). Only 3 patients experienced CTCAE grade 3 fatigue. No other patients experienced any $\geq$ CTCAE grade 3 toxicity. This analysis reports the result of a novel RT delivery technique for the treatment of patients with recurrent brain metastases. Side effects were manageable and comparable to other conventional repeat WBRT series. Repeat WB-SIB using the VMAT RT delivery technology is feasible and appears to have acceptable short-term acute toxicity. These results may provide a foundation for further exploration of the WB-SIB technique for repeat WBRT in future prospective clinical trials.
\end{abstract}

\section{Introduction}

Metastases to the brain occur in $25-45 \%$ of cancer patients [1]. With improving systemic treatments, cancer death rates are decreasing and patients with metastatic disease are living longer [2]. Standard treatment for patients with unresectable brain metastasis includes whole brain radiation therapy (WBRT), stereotactic radiosurgery (SRS), or a combination of both $[3,4]$. Local and distant brain progression after conventional WBRT are common, occurring in $47-86 \%$ of patients [5]. Potential interventions after WBRT include surgical resection, chemotherapy, SRS, or repeat WBRT. The radiation dose given at the time of repeat WBRT generally is reduced secondary to toxicity concerns and ranges from 20 to $30 \mathrm{~Gy}$ [6-13]. This only provides limited biologic effect on gross disease, yet still carries a substantial risk for neurocognitive delay to the patient.

Stereotactic radiosurgery (SRS) has frequently been combined with WBRT to prevent intracranial disease progression [3]. The additional SRS can significantly improve OS in patients with 1 brain metastasis and improve palliation in patients with $\leq 3$ brain metastases [3]. Despite its benefits, SRS is generally contraindicated in larger lesions (e.g., $>3.5 \mathrm{~cm}$ ).

Recent advances in RT delivery have changed the manner in which WBRT can be delivered. Novel RT techniques, such as volumetric modulated arc therapy (VMAT), use as few as one 358-degree rotation of the linear accelerator to deliver highly conformal dose distributions. This can result in highly conformal RT plans and steep dose gradients outside of brain metastases [14]. 
Patients with metastatic brain disease treated with WBRT alone will still recur frequently. What should be the RT management of patients with intracranial progression after prior WBRT? The current options consist of best supportive care, repeat WBRT, surgical resection, chemotherapy, SRS, or a combination of these. Extrapolating from the local control (LC) benefits of dose escalation in the upfront management of brain metastases, we have used VMAT in the setting of progression after WBRT to deliver a lower dose of repeat WBRT, while simultaneously boosting gross disease (WBSIB). The objective of this technique is to spare the normal brain tissue from excessively high doses of radiation and focus the boost doses on the tumor location specifically. The purpose of this retrospective review was to describe the technique of repeat WB-SIB and report the OS, toxicity, and LC outcomes of the first 16 patients treated using this novel modality.

\section{Patients and Methods}

2.1. Patients. A total of 16 patients received a repeat course of WBRT between 2006 and 2010 using the WB-SIB technique. The majority had favorable characteristics, including Karnofsky Performance Status (KPS) $\geq 70 \%$, low extracranial disease burden, and recursive partitioning analysis (RPA) class I or II [15]. Clinical response to reirradiation was judged from the notes in the medical record made $\geq 30$ days after the completion of repeat WB-SIB. Changes in clinical status were according to the assessment of the attending physician at that time and classified as follows: improved improvement in neurological symptoms or KPS, stable unchanged neurologic exam or KPS, and progression decline in neurologic function or KPS. Acute toxicities were graded based on the review of individual weekly treatment check notes. Local failure was analyzed and defined as progression of gross disease that was previously included in the SIB volume. Distant disease progression was defined as the development of new metastasis outside of the previously treated volume. Progression after conventional WBRT was determined from official MRI reports along with imaging reviewed by the attending radiation oncologist (WC, IC, and HS). This study was approved by the Emory Institutional Review Board.

2.2. Treatment Technique. All patients underwent a high resolution treatment planning MRI scan with and without contrast prior to CT simulation. A high resolution, thin slice (0.625 mm slice thickness) planning CT scan (PCT) without contrast was obtained for treatment planning. Patients were immobilized using the Efficast high precision thermoplastic immobilization mask and baseplate system (Orfit Industries, Jericho, NY). The PCT and treatment planning MRI scans were registered using Velocity AI (Velocity Medical Systems, Atlanta, GA). The boost gross target volume (GTV) was defined as the enhancing intracranial lesion and did not include surrounding areas of edema. The boost planning target volume (PTV1) involved a GTV expansion between 1 and $3 \mathrm{~mm}$. The brain was subsequently contoured as a CTV and $2 \mathrm{~mm}$ was added to create the whole brain PTV
(PTV2). Once all contouring was completed in velocity, structures were exported to eclipse (Varian Medical Systems, Palo Alto, CA) for treatment planning. Prescription dose to PTV2 varied according to the attending physician with a range of 15-25 Gy over 5-10 fractions. Additionally, the SIB or PTV1 dose ranged from 25 to 40 Gy also delivered over 510 fractions. The radiation oncologist and medical physicist were involved in the creation of the treatment volumes, normal structure delineation, treatment planning, and plan review for all cases.

Patients receiving WB-SIB were planned using 2-3 arcs of up to 358 degrees. Collimators were rotated to either $45 / 315$ or 30/330 degrees and jaws for each arc were opened up to accommodate the entire whole brain PTV volume. During optimization, arc avoidance sectors were created to avoid delivering entrance dose through the left and right orbits. Dose gradients between PTV-2 (whole brain) and PTV-1 (SIB) were kept at a minimum during optimization.

Goals of treatment planning were to obtain 95\% PTV coverage with the prescription dose and, whenever possible, a minimum dose to the PTV of $90 \%$ of the prescribed dose. Normal tissue constraints were left to the discretion of the attending physician. Prior to the delivery of VMAT plans, rigorous dosimetric quality assurance was conducted using a chamber array (MatriXX from IBA) and Gafchromic film. Preceding treatment delivery, each patient was set up to the marks on their immobilization mask using the room lasers. Orthogonal $\mathrm{kV}$ images were taken and matched to their respective DRRs utilizing Varian 4D online review system with adjustments to the patient's set-up position made in the lateral, longitudinal, and vertical directions prior to treatment. Many patients also received a cone beam CT with 4 degrees of freedom alignment (3 translation shifts and couch rotation) matching prior to treatment.

For assessing patterns of local failure, the planning MRI scan and DICOM RT dose files were registered to the diagnostic high resolution postcontrast MRI scan demonstrating local failure using Velocity AI. In patients that progressed after repeat WB-SIB, the sites of disease progression were recorded as either local (progression of a previously boosted lesion) or distant (new foci outside of the SIB dose distribution).

2.3. Statistical Analysis. Descriptive statistics were compiled to characterize the patient population. The Kaplan-Meier (KM) product-limit method was used for determining overall survival (OS). Time-to-event was defined from the end of repeat WB-SIB in all cases. For OS, events were death from any cause with censoring at time of last follow-up. All analyses were carried out using the SPSS version 19.0 statistical software package (IBM Inc., Armonk, NY).

\section{Results}

3.1. Patient Characteristics. Thirty-nine patients were identified that had undergone a course of brain irradiation using the WB-SIB boost technique from 2006 to 2010. Of these, 8 received upfront WB-SIB, 5 were treated for primary brain 
TABLE 1: Patient characteristics $(N=16)$.

\begin{tabular}{|c|c|}
\hline Age & $55(24-73)$ \\
\hline \multicolumn{2}{|l|}{ Sex } \\
\hline Male & 7 \\
\hline Female & 9 \\
\hline \multicolumn{2}{|l|}{ Race } \\
\hline White & 11 \\
\hline $\mathrm{AA}$ & 5 \\
\hline \multicolumn{2}{|l|}{ Primary histology } \\
\hline NSCLC & 5 \\
\hline Breast & 5 \\
\hline Small cell lung & 4 \\
\hline Melanoma & 1 \\
\hline Adenocarcinoma & 1 \\
\hline \multicolumn{2}{|l|}{ KPS (at time of repeat WB-SIB) } \\
\hline 90 & 5 \\
\hline 80 & 5 \\
\hline 70 & 1 \\
\hline$<70$ & 5 \\
\hline \multicolumn{2}{|l|}{ RPA class (at time of repeat WB-SIB) } \\
\hline $\mathrm{I}$ & 3 \\
\hline II & 8 \\
\hline III & 5 \\
\hline Median Graded Prognostic Index (GPA) & 1 \\
\hline Median number of lesions (at time of repeat WB-SIB) & $9(7-47)$ \\
\hline Total number of boosted lesions & 202 \\
\hline Use of steroids during the course of repeat WB-SIB & $16 / 16$ \\
\hline Median PTV volume (for repeat WB-SIB) & $21.84 \mathrm{~cm}^{3}(3.66-236.2)$ \\
\hline Median time from initial WBRT to repeat WB-SIB (days) & $339.5(97-1895)$ \\
\hline \multicolumn{2}{|l|}{ Systemic disease status at time of repeat WB-SIB } \\
\hline Progressive & 3 \\
\hline Stable & 13 \\
\hline Median clinical follow-up & 77.0 days \\
\hline \multicolumn{2}{|l|}{ Clinical outcome } \\
\hline Improved & 7 \\
\hline Stable & 6 \\
\hline Declined & 2 \\
\hline Median imaging follow-up & 42.5 days \\
\hline
\end{tabular}

WB-SIB: whole brain simultaneous infield boost, KPS: Karnofsky Performance Status, WBRT: whole brain radiation therapy, OS: overall survival, RPA: recursive partitioning analysis, and NSCLC: non-small-cell lung cancer.

tumors, and 10 pediatric patients were excluded. Median number of brain metastases at the time of initial WBRT was 3.5 (range: $0-17)$. Two patients initially underwent prophylactic cranial irradiation (PCI) and did not have any gross lesions at the time of their initial WBRT. Median number of brain metastases at the time of repeat WB-SIB was 9 (range: 7-47). Breast [5] and non-small-cell lung cancer (NSCLC) [5] were the most common histologies, followed by small cell lung cancer [4], melanoma [1], and adenocarcinoma of unknown primary [1]. The Graded Prognostic Assessment (GPA) of our patients was calculated using the method previously described [16]. See Table 1 for additional patient characteristics.
3.2. Treatment Characteristics. Median dose of the initial course of conventionally delivered WBRT was 35 Gy over 14 fractions (range: 25-50.4 Gy). Median time interval between the initial WBRT and repeat WB-SIB was 11.3 months. Median dose for repeat WB-SIB was $20 \mathrm{~Gy}$ to the whole brain with a median SIB boost dose of $30 \mathrm{~Gy}$. The range of dose for the repeat WB-SIB to the whole brain PTV was 15-25 Gy given over 5-10 fractions and with an SIB dose to gross disease of 25-40 Gy also given over 5-10 fractions. Expressed as $2 \mathrm{~Gy}$ equivalent fractionation schedules, the whole brain received approximately $18-28 \mathrm{~Gy}$ and gross lesions received approximately $30-46 \mathrm{~Gy}$ (assuming $\alpha / \beta$ of 2 and 10 , resp.). Of the 16 analyzable patients, 5 had SRS 
(range: $1-4$ treatments) in addition to WBRT before receiving repeat WB-SIB. Median PTV-1 volume for the WB-SIB treatment was $21.84 \mathrm{cubic} \mathrm{cm}$. Use of surgery was limited in this series with only one patient undergoing resection after the WB-SIB treatment with the final pathology of this specimen consistent with radiation necrosis. No patients underwent surgical interventional prior to the WB-SIB.

3.3. Follow-Up and Toxicity. Clinical follow-up data was available in 15/16 patients; 1 patient was lost to follow-up after completion of treatment. Of the remaining 15 patients, median clinical follow-up period was 77 days (range: 9-425). Only 3 patients remain alive at last follow-up with follow-ups between 7 and 10 months. Imaging follow-up was available in 13/16 patients with at least one follow-up MRI scan after completing their treatment with a median imaging follow-up period of 35.5 days (range: $0-425$ ).

Although acute toxicity (occurring less than 90 days from the start of treatment) was relatively common, they tended to be minor and consisted primarily of fatigue, which was experienced by $81 \%$ of patients. Only 3 patients experienced Common Terminology Criteria for Adverse Events (CTCAE) toxicity greater than grade 2 (all were grade 3 fatigue). The range of toxicities was what would be expected in patients receiving repeat WBRT. Table 2 summarizes these toxicities.

Late toxicity was difficult to assess given the retrospective nature of the study along with the short OS and limited follow-up. Out of 162 lesions boosted with the SIB technique that had at least one follow-up MRI, there was only one documented instance of radionecrosis that was surgically resected.

3.4. Local Control and Clinical Outcomes. Following repeat WB-SIB treatment, only two patients demonstrated intracranial disease progression for a crude control rate of $84.6 \%$ (11/13) in those patients with available follow-up imaging. Of the patients that had intracranial control $72.7 \%(8 / 11)$ ultimately developed extracranial disease progression. In the patients with MRI follow-up, 161 of 162 treated lesions (99.4\%) were stable or smaller at the date of last MRI. The median interval to imaging in this group of patients was 42.5 days from completion of treatment. Of the two patients that developed intracranial progression, one progressed at a site outside of the SIB dose distribution (distant brain progression) without local progression. The second patient progressed in an area that was within the prior SIB boost dose as well as an additional new focus outside of the PTV-1 (local and distant brain progression).

Clinical follow-up data was available for 15 of 16 patients. In these 15 patients, 7 improved clinically, 6 remained stable, and 2 declined as of their last follow-up visit.

The median OS time from initial WBRT was 18.9 months (95\% CI: 0.813-36.987) (Figure 1). The median OS time from the initiation of the time of repeat WB-SIB was 2.73 months (range: 0.46-14.46 (95\% CI: 1.3-8.7)) (Figure 2). Upon further exploratory subgroup analysis, patients were excluded with histologies known to carry a poor prognosis
TABLE 2: Acute side effects.

\begin{tabular}{lccc}
\hline & $N$ & $\begin{array}{c}\text { CTCAE grade } \\
(n \text { patients })\end{array}$ & Overall rate \\
\hline Any report & 15 & & $93.8 \%$ \\
Fatigue & 13 & $3(3)$ & $81 \%$ \\
& & $2(2)$ & \\
Anorexia & 2 & $2(1)$ & $12.5 \%$ \\
Nausea/vomiting & 1 & $1(1)$ & \\
Mood alteration & 1 & $2(1)$ & $6.25 \%$ \\
Radiation & 1 & 2 & $6.25 \%$ \\
dermatitis & & 1 & $6.25 \%$ \\
Motor & 2 & $2(2)$ & $12.5 \%$ \\
None & 2 & $\mathrm{n} / \mathrm{a}$ & $12.5 \%$ \\
\hline
\end{tabular}

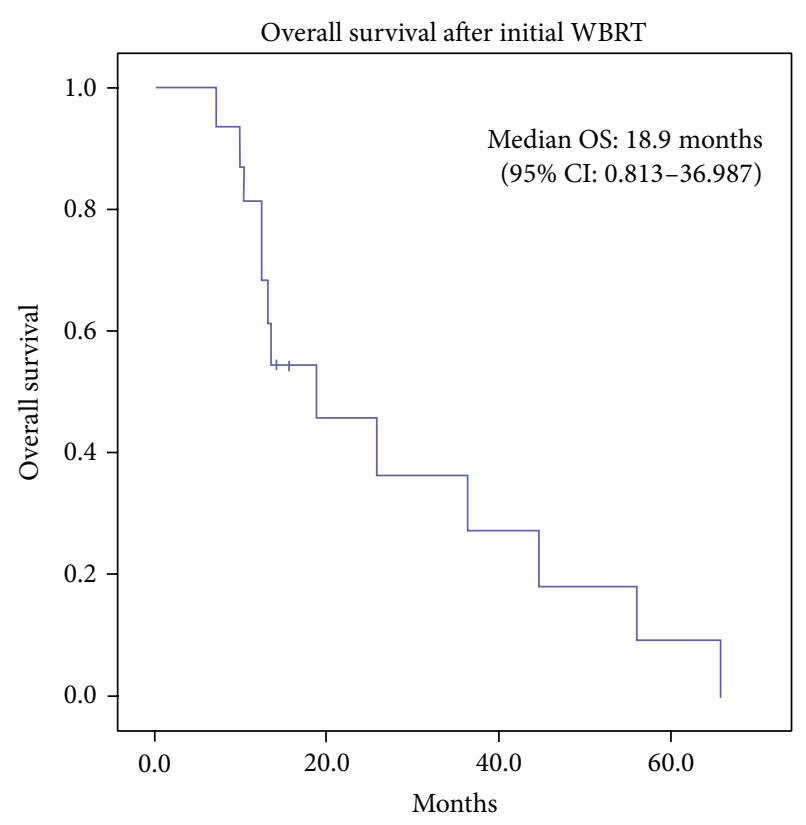

FIGURE 1: KM curve demonstrating OS after initial WBRT.

(melanoma and small cell lung cancer, $n=5$ ), and median OS was found to be 6.7 months (range: 0.46-14.4) (Table 3).

\section{Discussion}

Dose escalation of gross disease in the brain with SRS in addition to WBRT clearly improves OS for patients with solitary metastasis and LC/palliation in patients with up to 3 metastases [3]. For patients with $\geq 4$ metastases, treatment with WBRT provides useful palliation [17]. Recently, the application of the novel technology of VMAT for selective dose escalation in patients with multiple metastatic lesions has been presented in the upfront setting of multiple brain metastases [14]. The application of this novel technique for 


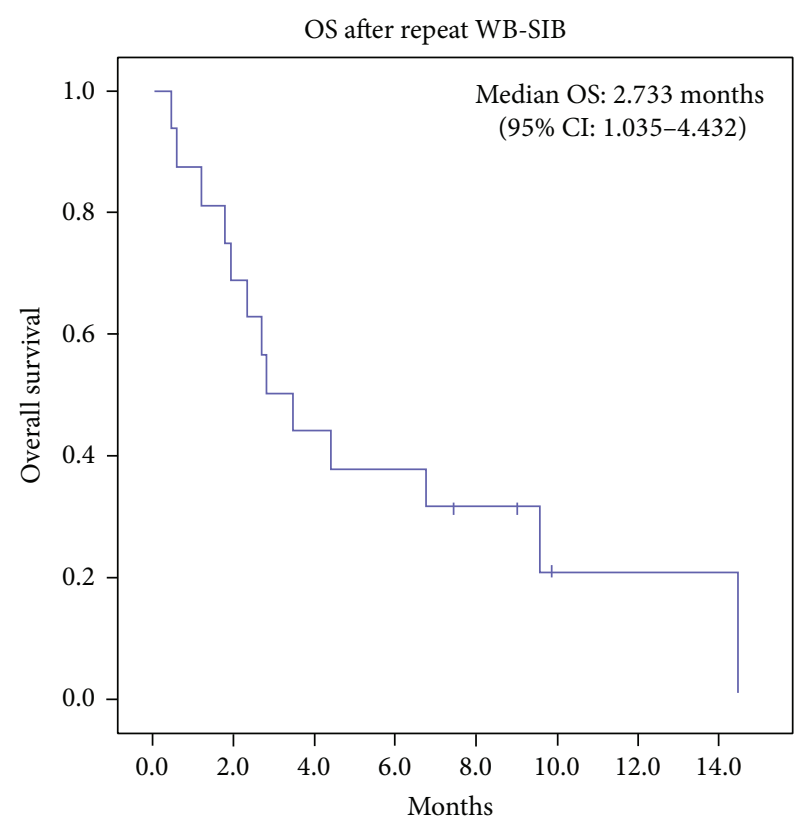

FIgURE 2: KM curve showing OS after repeat WB-SIB.

TABLE 3: Subgroup analysis, excluding small cell and melanoma $(n=$ 11).

\begin{tabular}{lc}
\hline OS & $\begin{array}{c}\text { 202 days (14-434) } \\
\text { NSCLC, breast, and } \\
\text { adenocarcinoma }\end{array}$ \\
Histology & 52 \\
RPA class & I-2 \\
& II-6 \\
KPS & III-3 \\
Median time from initial WBRT & $90 \%$ \\
to repeat WB-SIB & 553.5 days \\
\hline
\end{tabular}

WB-SIB: whole brain simultaneous infield boost, KPS: Karnofsky Performance Status, WBRT: whole brain radiation therapy, OS: overall survival, and RPA: recursive partitioning analysis.

the treatment of the whole brain is exciting and may carry promise for further evaluation.

Unlike upfront management of brain metastases patients, the literature is relatively limited for guiding treatment of patients with intracranial relapse after WBRT [6-13], despite its occurrence in $47-86 \%$ of patients [5]. Furthermore, recommendations are often conflicting, and the existing series span decades over which patient survival and RT delivery techniques have changed significantly [6-13]. With the increasing ability of chemotherapy to control extracranial disease, the role of effective intracranial control is likely to become of greater importance.

The application of the novel WB-SIB technique seeks to escalate dose to gross disease while sparing the normal brain from the toxicity of repeat WBRT to conventional doses. Because dose escalation (by SRS) is beneficial for patients with brain metastasis in the primary setting, our expectation is that reirradiation with the WB-SIB technique will improve LC and thus palliation. Furthermore, the ability of WB-SIB to reduce the dose to the normal brain parenchyma while at the same time escalating the radiation dose to the gross disease is an exciting application of this technology. Potential benefit of this technique is also supported by Wong et al's findings of improved outcomes for higher doses with repeat WBRT [10].

The crude LC rate in our series was $99.4 \%$, and while this number is limited by poor imaging follow-up, it is encouraging. An example of sequential MRI follow-ups with treatment response is shown (Figure 3). While retrospective assessment of palliative endpoints is difficult, the majority (86\%) of our patients had either stable or improved neurological status. This is comparable to other published series (Table 4).

While a median OS from the time of repeat RT of 2.7 months is low, it is comparable to most other reirradiation series (Table 4) [6-13]. Notwithstanding the substantial limitations of small retrospective subgroup analysis, we performed an exploratory subgroup analysis and eliminated patients with known poor prognostic histologies (melanomas and small cell lung cancers); the 11 remaining patients were found to have a crude median OS of 6.7 months (Table 3). These results may demonstrate that more stringent patient selection criteria are needed to fully realize the benefits of the WB-SIB technique. The time interval between initial and repeat irradiation may also be considered. In our series, the average time to reirradiation was 11.3 months. Although a study by Sadikov et al. concluded that this time interval did not have prognostic significance [11], we expect that longer intervals between initial WBRT and reirradiation would correlate with extracranial disease status, which has been shown to be prognostic in the reirradiation setting $[11,13]$.

Increasing number of brain metastases is known to be a poor prognostic factor with both initial and repeat WBRT $[10,16,18]$. Of the published series cited on repeat WBRT, the median number of brain metastases present at reirradiation is generally not reported, making accurate comparisons of OS times difficult. The median number of brain metastases in our series was 9 , with the fewest number being 7 . While it is not validated in the setting of repeat WBRT, in an attempt to estimate an expected OS for this cohort, the average GPA of our patients at the time of repeat WBRT treatment was calculated to be 1 . Had this been applied in the upfront WBRT setting, it would yield an estimated median OS of only 2.6 months [16]. As our patients were undergoing repeat treatment, we might expect even worse outcomes than would be seen in the upfront treatment. When considered in this light, our reported median OS time of 2.7 months may actually suggest better results than might be expected. However, this extrapolation of OS comparison is subject to multiple limitations and is really only presented for hypothesis generating purposes.

The rates of acute toxicity in our series (93.7\%) were slightly higher than those reported in other major series [6-13]. While the exact reason for this discrepancy is not known, differences in the definition of acute side effects and/or how they are recorded are likely factors. In our series, weekly treatment check notes were reviewed individually for each patient to determine the CTCAE acute toxicity grading. 
TABLE 4: Literature summary.

\begin{tabular}{|c|c|c|c|c|c|c|c|c|c|}
\hline & $\begin{array}{l}\text { Shehata } \\
\text { et al. [6] }\end{array}$ & $\begin{array}{l}\text { Kurup } \\
\text { et al. [7] }\end{array}$ & $\begin{array}{c}\text { Hazuka } \\
\text { and Kinzie } \\
{[8]} \\
\end{array}$ & $\begin{array}{l}\text { Cooper } \\
\text { et al. [9] }\end{array}$ & $\begin{array}{c}\text { Wong } \\
\text { et al. [10] }\end{array}$ & $\begin{array}{l}\text { Abdel- } \\
\text { Wahab } \\
\text { et al. [12] }\end{array}$ & $\begin{array}{l}\text { Sadikov } \\
\text { et al. [11] }\end{array}$ & $\begin{array}{c}\text { Son } \\
\text { et al. [13] }\end{array}$ & $\begin{array}{c}\text { Present } \\
\text { study }\end{array}$ \\
\hline Number of patients & 35 & 56 & 44 & 52 & 86 & 15 & 72 & 17 & 16 \\
\hline $\begin{array}{l}\text { Initial RT } \\
\text { fractionation } \\
\text { (dose Gy/num frac) }\end{array}$ & $\begin{array}{l}35 / 10 \\
30 / 10\end{array}$ & $\begin{array}{c}18 / 3 \\
20 / 5 \\
30 / 10\end{array}$ & $30 / 10$ & $30 / 10$ & $30 / 10$ & $35 / 10$ & $20 / 5$ & $35 / 14$ & $35 / 14$ \\
\hline $\begin{array}{l}\text { Repeat WBRT } \\
\text { fractionation } \\
\text { (dose Gy/num frac) }\end{array}$ & $10 / 1$ & $20 / 10$ & $25 / 8$ & $25 / 10$ & $20 / 10$ & $\begin{array}{l}\text { 30/20 BID } \\
\text { (partial } \\
\text { brain) }\end{array}$ & $25 / 10$ & $21.6 / 12$ & $\begin{array}{l}\text { 20/10 WB } \\
30 / 10 \mathrm{SIB} \\
\text { boost }\end{array}$ \\
\hline $\begin{array}{l}\text { Time between initial } \\
\text { and repeat WBRT } \\
\text { (months) }\end{array}$ & NR & 6.3 & 7.8 & $>4$ & 7.6 & 10 & 9.6 & 15 & 11.3 \\
\hline \multicolumn{10}{|l|}{ Response } \\
\hline Improved & $68 \%$ & $75 \%$ & $27 \%$ & $42 \%$ & $70 \%$ & $60 \%$ & $40 \%$ & $80 \%$ & $46 \%$ \\
\hline Stable & $25 \%$ & $12.5 \%$ & $41 \%$ & $52 \%$ & $29 \%$ & $27 \%$ & $33 \%$ & $20 \%$ & $40 \%$ \\
\hline Survival (months) & NR & 3.5 & 2 & 4 & 4 & 3.2 & 4.1 & 5.2 & 2.7 \\
\hline $\begin{array}{l}\text { Percentage of patients } \\
\text { with solitary lesion at } \\
\text { the time of repeat } \\
\text { WBRT }\end{array}$ & NR & NR & NR & NR & $45 \%$ & NR & $14 \%$ & NR & $0 \%$ \\
\hline
\end{tabular}

NR: not reported, WBRT: whole brain radiation therapy, Gy: gray, Num: number, and Frac: fractions. Table is adapted from that presented by Sadikov et al. and Son et al. [11, 13].

Time of repeat WB-SIB

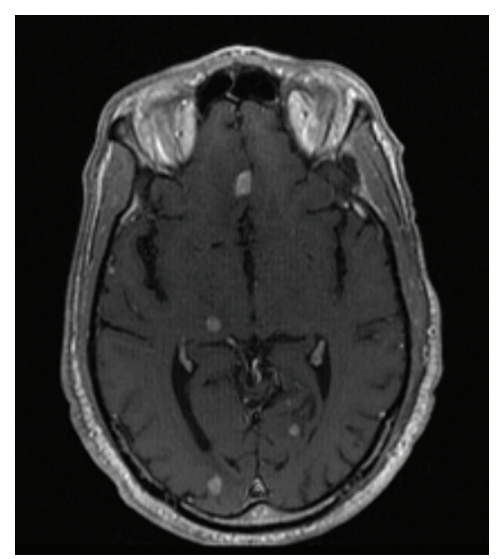

(a)
3 month

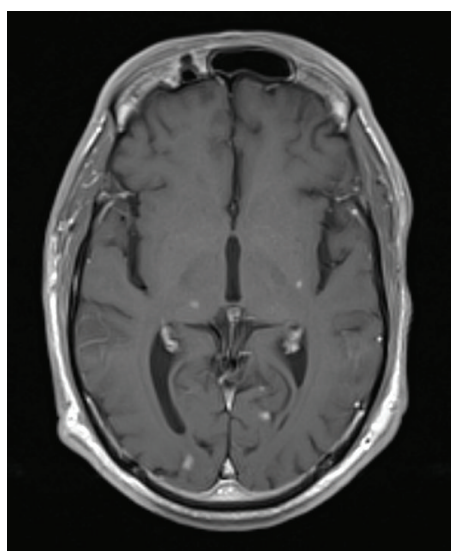

(b)
6 month

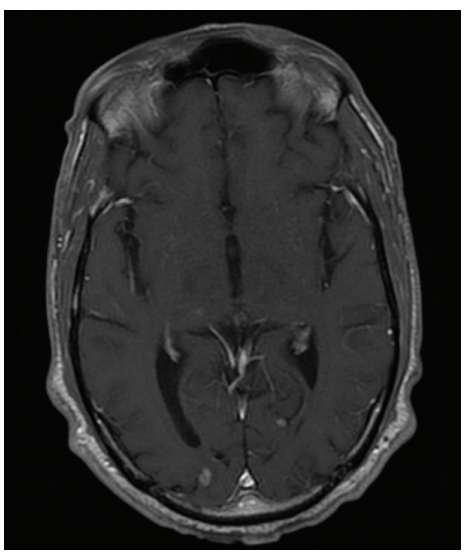

(c)

FIGURE 3: Example of sequential MRIs after repeat WB-SIB treatment.

It is important to note that the reported acute toxicities were typically mild and manageable and consisted mainly of fatigue. In fact, only 3 patients experienced $>$ CTCAE grade 2 toxicity and this consisted, in each instance, of grade 3 fatigue. Late toxicity was difficult to assess given the limited followup time. Only one instance of radionecrosis requiring surgery was noted in this cohort. While the total number of patients was small, it is important to consider that the total number of boosted lesions with imaging follow-up in this series was 162 , with only one documented instance of radiation necrosis.
This current series needs to be interpreted with caution due to several limitations. It is highly probable that patient selection played a large role in the OS outcomes presented. Additionally, there is substantial patient heterogeneity. We have included two patients treated with prophylactic RT for small cell lung cancer, along with one patient with melanoma. The outcome for the single patient with melanoma was very poor with rapid progression after the course of WB-SIB [19]. Additionally, the patients with small cell lung cancer had an average OS after repeat WB-SIB of only 50 days, likely 
a consequence of the aggressive natural history of small cell lung cancer. Better patient selection could have resulted in better patient outcomes.

One case in our cohort, while anecdotal, illustrates the potential benefits of therapy. This subject initially received WBRT for multiple brain metastases from breast cancer. She required salvage SRS at 3 different sites over the ensuing 4 years until she presented with multiple new lesions in the brain ( $>10$ metastases). The patient was treated with 15 Gy to the whole brain with a 25 Gy SIB dose to visible metastatic lesions in 5 fractions. Intracranial control was achieved until she succumbed to widespread extracranial metastatic disease 14 months later, never having developed intracranial progression.

The results of this series are intended to be hypothesis generating only and to introduce the feasibility and novel application of VMAT in the management of patients with progressing brain metastases. It is difficult to make firm recommendations for an optimal dose and fractionation schedule based on this small retrospective series. Moreover, the ideal dosimetric delivery of radiation for these patients remains unclear and requires additional investigation. If such patients are treated outside the setting of a clinical trial at our institution they are typically treated to 20 Gy over 10 fractions to the whole brain with an SIB dose of $30 \mathrm{~Gy}$ to gross disease. Ideally, patients in whom this technique is being considered would be entered into a prospective clinical trial.

\section{Conclusions}

We have been able to demonstrate the feasibility of a novel RT delivery technologic application for purposes of repeat WBRT. The OS and acute toxicity results presented appear to be comparable to other recently published series using conventional repeat WBRT; however, limitations to our analysis do exist. Only a small amount of data on the management of patients who have failed WBRT exists, and there is very limited available data on using novel RT delivery technologies in the repeat WBRT setting. This retrospective analysis may provide a foundation for further investigation of the technologic advancement of WB-SIB for repeat WBRT. While the WB-SIB technique is not appropriate for every patient who has failed WBRT, the feasibility and potential benefits of this technique in selected patients warrant further investigation in prospective clinical trials.

\section{Conflict of Interests}

Ian Crocker receives royalties derived from Velocity Medical Solution's sale of products. The terms of this agreement have been reviewed and approved by Emory University in accordance with its conflict of interests policies.

\section{References}

[1] E. S. Nussbaum, H. R. Djalilian, K. H. Cho, and W. A. Hall, "Brain metastases. Histology, multiplicity, surgery, and survival," Cancer, vol. 78, no. 8, pp. 1781-1788, 1996.
[2] A. Jemal, R. Siegel, J. Xu, and E. Ward, "Cancer statistics, 2010," CA: A Cancer Journal for Clinicians, vol. 60, no. 5, pp. 277-300, 2010.

[3] D. W. Andrews, C. B. Scott, P. W. Sperduto et al., "Whole brain radiation therapy with or without stereotactic radiosurgery boost for patients with one to three brain metastases: phase III results of the RTOG 9508 randomised trial," The Lancet, vol. 363, no. 9422, pp. 1665-1672, 2004.

[4] H. Aoyama, H. Shirato, M. Tago et al., "Stereotactic radiosurgery plus whole-brain radiation therapy vs stereotactic radiosurgery alone for treatment of brain metastases: a randomized controlled trial," Journal of the American Medical Association, vol. 295, no. 21, pp. 2483-2491, 2006.

[5] B. Borgelt, R. Gelber, and S. Kramer, "The palliation of brain metastases: final results of the first two studies by the radiation therapy oncology group," International Journal of Radiation Oncology*Biology*Physics, vol. 6, no. 1, pp. 1-9, 1980.

[6] W. M. Shehata, F. R. Hendrickson, and W. A. Hindo, "Rapid fractionation technique and retreatment of cerebral metastases by irradiation," Cancer, vol. 34, no. 2, pp. 257-261, 1974.

[7] P. Kurup, S. Reddy, and F. R. Hendrickson, "Results of reirradiation for cerebral metastases," Cancer, vol. 46, no. 12, pp. 2587-2589, 1980.

[8] M. B. Hazuka and J. J. Kinzie, "Brain metastases: results and effects of re-irradiation," International Journal of Radiation Oncology*Biology*Physics, vol. 15, no. 2, pp. 433-437, 1988.

[9] J. S. Cooper, A. D. Steinfeld, and I. A. Lerch, "Cerebral metastases: value of reirradiation in selected patients," Radiology, vol. 174, no. 3, pp. 883-885, 1990.

[10] W. W. Wong, S. E. Schild, T. E. Sawyer, and E. G. Shaw, "Analysis of outcome in patients reirradiated for brain metastases," International Journal of Radiation Oncology*Biology*Physics, vol. 34, no. 3, pp. 585-590, 1996.

[11] E. Sadikov, A. Bezjak, Q.-L. Yi et al., "Value of whole brain re-irradiation for brain metastases-single centre experience," Clinical Oncology, vol. 19, no. 7, pp. 532-538, 2007.

[12] M. M. R. Abdel-Wahab, A. H. Wolfson, W. Raub et al., "The role of hyperfractionated re-irradiation in metastatic brain disease: a single institutional trial," American Journal of Clinical Oncology: Cancer Clinical Trials, vol. 20, no. 2, pp. 158-160, 1997.

[13] C. H. Son, R. Jimenez, A. Niemierko, J. S. Loeffler, K. S. Oh, and H. A. Shih, "Outcomes after whole brain reirradiation in patients with brain metastases," International Journal of Radiation Oncology*Biology*Physics, vol. 82, 2, pp. e167-e172, 2011.

[14] F. J. Lagerwaard, E. A. P. van der Hoorn, W. F. A. R. Verbakel, C. J. A. Haasbeek, B. J. Slotman, and S. Senan, "Whole-brain radiotherapy with simultaneous integrated boost to multiple brain metastases using volumetric modulated arc therapy," International Journal of Radiation Oncology*Biology*Physics, vol. 75, no. 1, pp. 253-259, 2009.

[15] L. Gaspar, C. Scott, M. Rotman et al., "Recursive Partitioning Analysis (RPA) of prognostic factors in three Radiation Therapy Oncology Group (RTOG) brain metastases trials," International Journal of Radiation Oncology*Biology*Physics, vol. 37, no. 4, pp. 745-751, 1997.

[16] P. W. Sperduto, B. Berkey, L. E. Gaspar, M. Mehta, and W. Curran, "A new prognostic index and comparison to three other indices for patients with brain metastases: an analysis of 1,960 patients in the RTOG database," International Journal of Radiation Oncology*Biology*Physics, vol. 70, no. 2, pp. 510-514, 2008. 
[17] D. Kondziolka, A. Patel, L. D. Lunsford, A. Kassam, and J. C. Flickinger, "Stereotactic radiosurgery plus whole brain radiotherapy versus radiotherapy alone for patients with multiple brain metastases," International Journal of Radiation Oncology*Biology*Physics, vol. 45, no. 2, pp. 427-434, 1999.

[18] P. W. Sperduto, S. T. Chao, P. K. Sneed et al., "Diagnosisspecific prognostic factors, indexes, and treatment outcomes for patients with newly diagnosed brain metastases: a multiinstitutional analysis of 4,259 patients," International Journal of Radiation Oncology*Biology*Physics, vol. 77, no. 3, pp. 655-661, 2010.

[19] S. M. Sawrie, B. L. Guthrie, S. A. Spencer et al., "Predictors of distant brain recurrence for patients with newly diagnosed brain metastases treated with stereotactic radiosurgery alone," International Journal of Radiation Oncology*Biology*Physics, vol. 70, no. 1, pp. 181-186, 2008. 


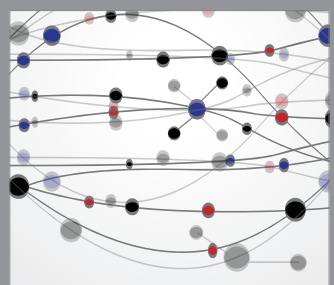

The Scientific World Journal
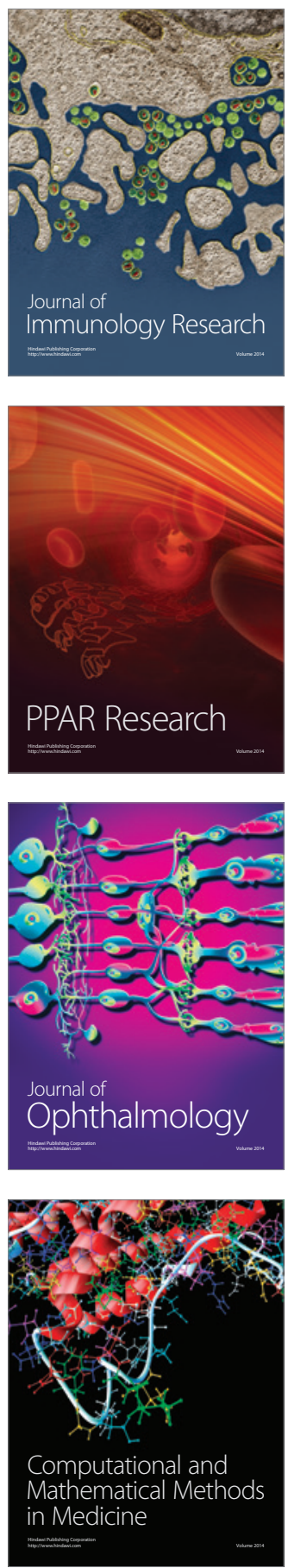

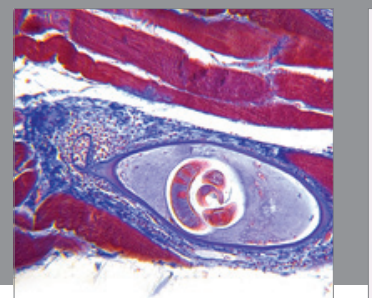

Gastroenterology

Research and Practice
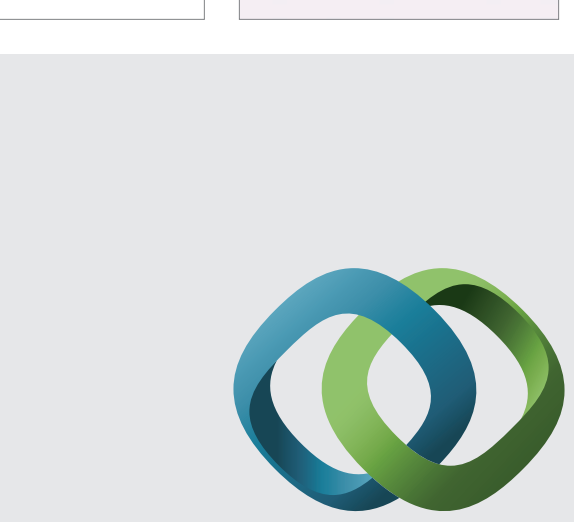

\section{Hindawi}

Submit your manuscripts at

http://www.hindawi.com
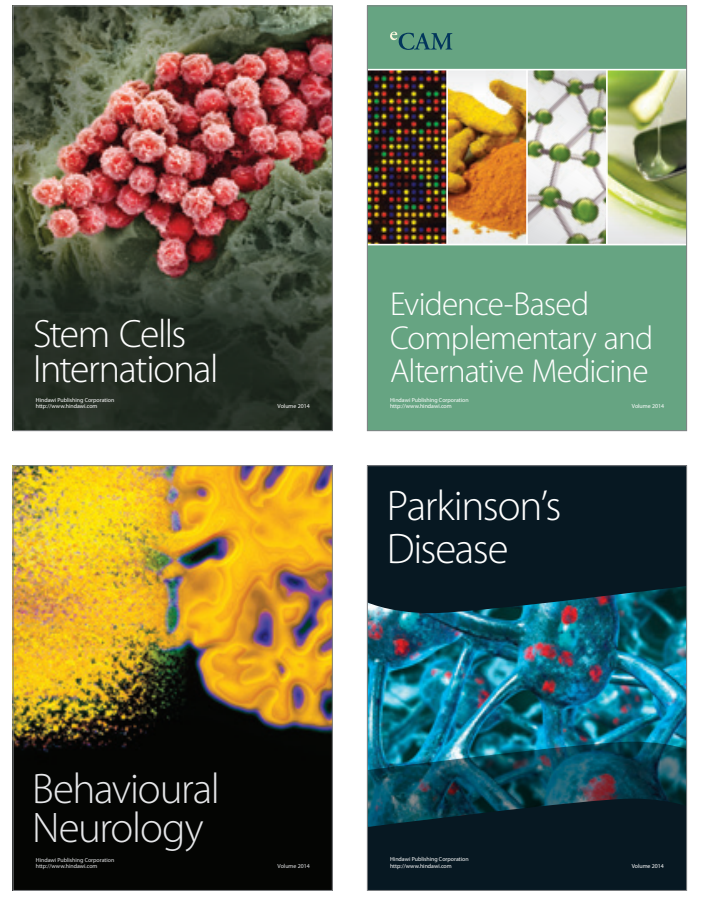
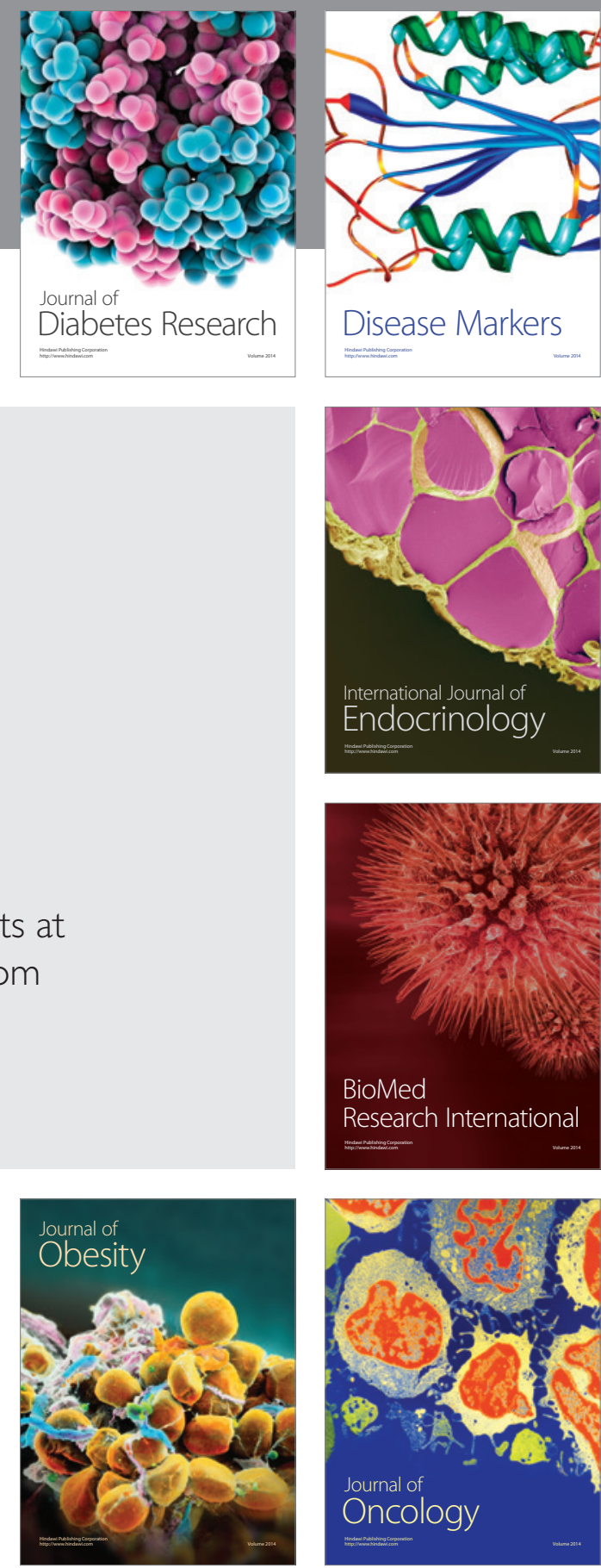

Disease Markers
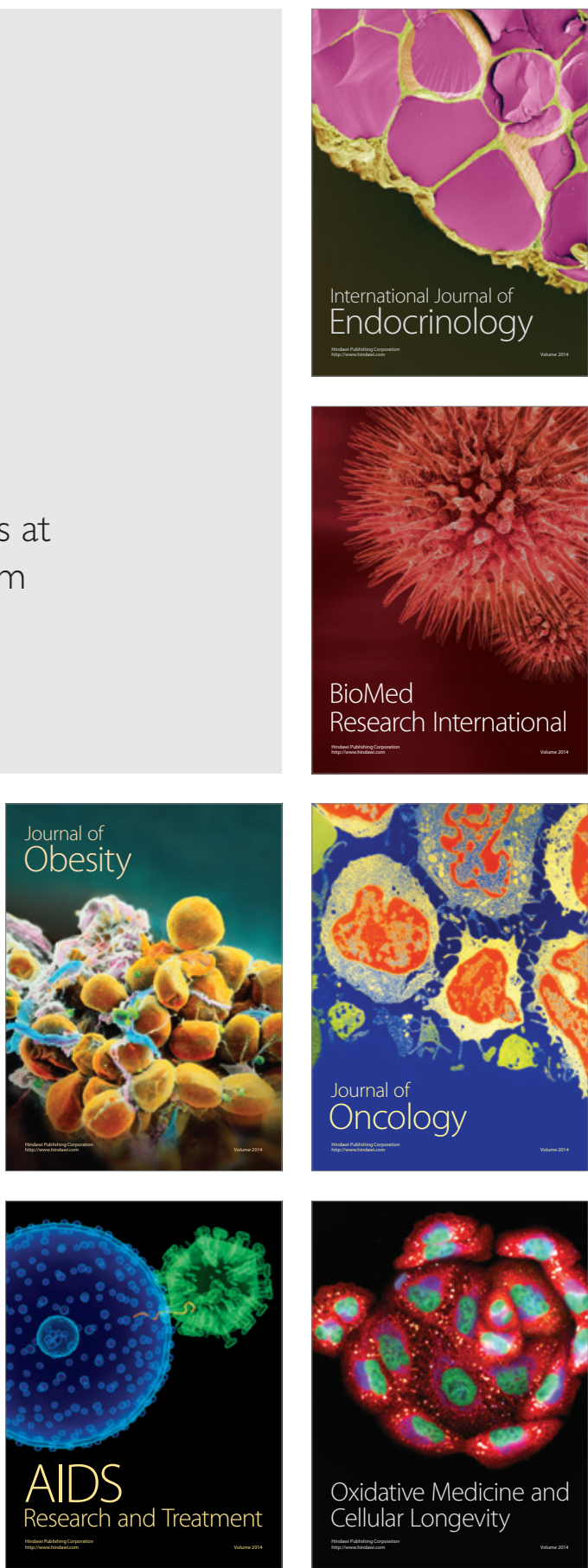